Research Article

\title{
Food Safety Practice and Associated Factors among Meat Handlers in Gondar Town: A Cross-Sectional Study
}

\author{
Dawit Getachew Yenealem $\mathbb{D}^{1},{ }^{1}$ Walelegn Worku Yallew $\mathbb{D}^{1}{ }^{1}$ and Shafi Abdulmajid ${ }^{1,2}$ \\ ${ }^{1}$ Department of Environmental and Occupational Health and Safety, Institute of Public Health, \\ College of Medicine and Health Sciences, University of Gondar, Gondar, Ethiopia \\ ${ }^{2}$ Department of Public Health, Eastern African Collage, Harrar Campus, Harrar, Ethiopia \\ Correspondence should be addressed to Dawit Getachew Yenealem; dawitgetachew105@gmail.com
}

Received 18 October 2019; Revised 15 January 2020; Accepted 4 February 2020; Published 24 February 2020

Academic Editor: Ping Xiang

Copyright (c) 2020 Dawit Getachew Yenealem et al. This is an open access article distributed under the Creative Commons Attribution License, which permits unrestricted use, distribution, and reproduction in any medium, provided the original work is properly cited.

\begin{abstract}
Introduction. Animal sources of foods (ASF), including meat, are a source of high-quality nutrients. However, meat composition makes it an ideal medium for the growth of a good number of microorganisms. Around 600 million foodborne illnesses and 420000 deaths occur each year due to poor food handling practice. Thus, probing into meat handling practice will be an insatiable input for the intervention. This study aims to investigate the level of meat handling practice and associated factors among meat handlers in butcheries in Gondar town, Ethiopia. Method. The study was a community-based cross-sectional study among butcher shops in Gondar town from April 20 to 30, 2019. Data were collected using a pretested structured questionnaire by trained data collectors among 214 meat handlers from butcher shops. Multivariable logistic regression analysis with a $95 \%$ confidence interval (CI) was used to identify the factors significantly associated with a good level of meat handling practice. Result. More than half of the meat handlers $66.4 \%$ (95\% CI: $(59.8,72.4)$ ) in butcher shops had a good level of meat handling practice. Level of attitude $(\mathrm{AOR}=4.45 ; 95 \% \mathrm{CI}, 2.09-9.43)$ and knowledge ( $\mathrm{AOR}=2.04 ; 95 \% \mathrm{CI}, 1.09-3.82)$ were significantly associated with a good level of meat handling practice. The majority of respondents wash their hands after disposing garbage (91.6\%) with less vigilance after smoking, sneezing, or coughing (64.0\%). Conclusion. The study revealed that the level of food handling practice was unsatisfactory among meat handlers. This result is a testimony to the prevailing potential risk faced by consumers due to the disregarding of hygienic behaviors by food handlers. Considering attitude and knowledge are associated with the outcome variable, investing time on behavioral change activities that will contribute to the improvement of meat handler's attitude \& practice, primarily focusing on reducing working while ill is essential. Therefore, much supervisory and coaching work will be expected from local health departments \& regulatory bodies.
\end{abstract}

\section{Introduction}

Most fresh food, particularly those from animals, are highly vulnerable to microbial contamination and food poisoning [1]. Meat composition makes it an ideal medium for the growth of a good number of microorganisms [2] due to richness in nutrients [3]. The majority of foodborne diseases arise from the food of animal origin $[4,5]$. The food handler's health status and hygiene practice are the foremost determinants of food contamination [6]. Food poisoning happens as a result of ingesting food contaminated with microorganisms or their toxins, the contamination springing up from insufficient protection methods, unhygienic dealing with practices, cross-contamination from food contact surfaces, or men and women harboring microbes [7]. This can result in quality deterioration and, hence, quantity losses, economic losses, and public health concerns $[8,9]$.

According to the World Health Organization, almost 1 in 10 people fall ill, and 420000 die every year, dining on cuisine tainted by microorganisms [10]. Other reports depicted that around 600 million foodborne illnesses and 420,000 deaths occur each year due to poor food handling practice [11], in which substantial proportion goes to meat- 
related hazards [12].In the Netherlands, About 53\% of the foodborne burden and $31 \%$ of all food-related cases were associated with meat [12]. A World Bank study finds that the impact of unsafe food costs low- and middle-income economies, nearly US\$ 110 billion in decreasing productivity and medical expenses each year [13]. One Ethiopian study claims that contamination of beef while transferring from the abattoir to the butcher shops with the highest source of contamination attributed to abattoir workers [14]. Foodborne diseases have been increasing in recent years, with an enormous impact on the health and economy of developing countries than those of developed countries [7, 15]. The impediment of socioeconomic development has manifested this by straining the health-care system and harming national economies, tourism, and trade [16].

Food handling and hygiene have been a great concern across industries [17]. Foods can be mishandled during preparation, processing, or storage [18]. It has been shown that most outbreaks of food poisoning result from improper food handling practices [19] that implicate the food handlers [20]. According to the Codex Alimentarius Commission, improper food handling is a major cause of foodborne diseases, and poor hand hygiene is a significant risk factor in the occurrence of food contamination [21]. Mishandling food supposed to be implicated in $97 \%$ of all food-borne illnesses associated with food service establishments [22]. European Food Safety Authority [23] reports that around $48.7 \%$ of foodborne illnesses are associated with food services in the food premises [21]. This was underlined by the fact that mishandling [24], worker health and hygiene [25], and the presence of Escherichia coli and Staphylococcus aureus on the hands of food handlers [26] were stated as specific reasons in studies. In developing countries, Foodborne diseases occur because of poor food handling and sanitation problems [27]. Food workers in many settings have been responsible for food borne disease outbreaks [28], and there is no indication that this is diminishing [19].

Thus, probing in handling practice of meat handlers will be an insatiable input for the intervention. Three factors are playing a fundamental role in food poisoning outbreaks concerning food handlers: knowledge, attitude, and practice [29]. Because meat is a highly perishable food, the knowledge and level of training of meat handlers in the meat industry in hygiene and food safety are of particular importance in ensuring the health and safety of the consumer [30]. Practices will render antecedently uncontaminated foods unsafe to eat, e.g., through cross contamination, and contaminated foods safe to eat, e.g., through thorough preparation [31]. The informal methods of meat handling and marketing meat by butcheries undermine meat quality and safety [32], which is shared by the similar socioeconomic conditions of Ethiopia. Customarily, food is sold and displayed in open shops without proper guarding [33], and cold-chain process [14] of hanging raw meat on the hooks and offering for consumption to consumers were followed in butcheries. Food-borne disease outbreaks remain a major global health challenge, and cross contamination from raw meat due to poor handling is a major cause in developing countries [19]. In Ethiopia, despite rising prices, the demand for meat products is dramatically increasing from $7.0 \mathrm{~kg}$ in 2000 to $8.5 \mathrm{~kg}$ in 2009 [34]; and the consumption of raw meat becomes a symbol of status [7].This study aims to investigate the level of meat handling practice and associated factors among food handlers in butcheries across Gondar town. It will be a spring board for further inquires on the problem and valuable inputs for concerned regulatory, health promotion bodies.

\section{Methods}

2.1. Study Design, Setting, and Period. The study was a community-based cross-sectional study among butcher shops in Gondar town from April 2019. The town of Gondar is located north of Addis Abeba at $737 \mathrm{~km}$ and $265 \mathrm{~km}$ from the regional capital Bahir Dar. Gondar was founded in 1635 and located in the central Gondar Zone of the Amhara Region, and it is one of the tourist destination places which have been a seat of a government. According to the central statistical agency of Ethiopia, in 2017, Gondar projected to be home for 360,600 people [35]. Administratively, the town is divided into 12 administrative areas. During the study, there were reportedly about 146 butcher shops in Gondar town. Among them, 129 are Christian ones, and the rest are Islamic ones.

2.2. Inclusion and Exclusion Criteria. Meat handlers who have direct contact with meat and meat handling surfaces with one year experience were considered to study population. Furthermore, those who were randomly selected were a study population. Those meat handlers who are not well communicated due to any disability or illness and seriously ill workers were excluded from the study.

\subsection{Sample Size Determination and Sampling Procedure.} The sample size was determined using a single population proportion formula $n=Z^{2} p(1-p) / d^{2}$. Since similar studies in our country on this study subjects are lacking, we take $50 \%$ prevalence for meat handling practice with $95 \%$ confidence interval and margin of error $5 \%$ between the sample and the underlying population, which give us a sample size of 384. However, the final sample size was determined by using correction formula $F N=n / 1+n / N$ (since $N<10,000)$. By adding $10 \%$ nonresponse rate, the sample sizes became 224 .

The community-based cross-sectional study design was conducted. A systematic random sampling technique was used to select each butcher shop. Systematic random sampling was followed by proportional allocation for those groups of workers more than one member within butcher shops. Two hundred and fourteen meat handlers were selected from 12 subcities by systematic random sampling methods since we are unable to find a list of our subjects.

\subsection{Operational Definition}

2.4.1. Meat Handling Practice Level. The respondents who scored less than $70 \%$ of the correct answer of their response 
to 20 meat handling-related practice questions were considered as having "poor level of practice," and those who scored higher than or equal to $70 \%$ were considered as having "good practice level" [15].

2.4.2. Meat Handling Knowledge Level. The respondents who scored less than $70 \%$ of the correct answer of their response to 20 meat handling related knowledge questions were considered as having "poor level of knowledge" those who scored higher than or equal to $70 \%$ were considered as having "good level of knowledge" [7, 15, 20].

2.4.3. Meat Handling Attitude Level. The respondents who scored less than $70 \%$ of the correct answer of their response to 20 meat handling-related attitude questions were considered as having "poor level of attitude," and those who scored higher than or equal to $70 \%$ were considered as having "good level of attitude" [7, 15, 20].

2.5. Data Collection Tools and Procedures. Data were collected from meat handlers working in butcher shops in Gondar town. An interview-administered data collection technique was used to collect data. Two environmental health professionals were deployed for supervision, along with four environmental and occupational health and safety (EOHS) final year students as data collectors. The questionnaire was initially prepared in the English version, then translated to Amharic, and back to English to check consistency and clarity of the question. A pretested Interviewerbased structured questionnaire adapted from different literature $[7,15,19-21,24]$ to fit with both subject area, and local context was used to collect data in which a similar version was used in Ethiopia [20]. The questionnaires comprised four blocks of sections, namely: sociodemographic, knowledge, and attitude, and practice questions.

In the knowledge part, 20 close-ended questions were focusing on personal hygiene, cross contamination, microbiological food hazards of specific foodborne diseases, safe food handling, person-to-person food-borne disease transmission, and handwashing knowledge. Each question was followed by three potential responses (i.e., yes, no, and I don't know).

The subsequent part of the questionnaire was inquired about the attitudes of the responders by forwarding questions related to various hygienic measures for food safety. It comprises twenty questions where food handlers were asked to indicate their level of agreement to the statements using a three-point rating scale (i.e., I agree, I don't agree, and I don't know). The third option, "I don't know" has been introduced to facilitate ease of responding for participants by complimenting for thoughts characterized by an Undecidedness, uncertainty, or not aware of the inquiry at all.

The last section assesses practices of food workers by their selfreported hygienic behaviors. Similar numbers of questions were provided with Yes or No options. The items include eating or drinking at the workplace, washing hands: after handling waste, using the toilet, coughing or sneezing; habit of wearing gloves, masks \& aprons during work; routines during having illness and even presenting with cuts, wounds, bruises or injuries on hands; wear jewelry when handling meat. Response to part two to three treated as categorical variable considering correct answer as 1 and incorrect answer as 0 (including I don't know). In addition, a scale ranging between 0 and 20, which represents the total number of questions on the three sections were considered.

2.6. Data Quality Management and Analysis. All the questionnaires were checked visually, coded, and entered into Epi Info software version 7 and exported into SPSS version 20.0 for further analysis. Descriptive statistics were computed to summarize data, including frequenciesand percentages, and mean for each item of the questionnaire was presented by using graphs, tables, and charts. Moreover, tabulation and different forms of graphs were used to present the result. Bivariable and multivariable logistic regression analysis was conducted to identify factors associated with Meat handling practice. The strength of the associated factors was presented by the odds ratio with $95 \%$ CI. A $p$ value of less than 0.05 was considered statistically significant. Compiled results were presented in the form of text, tables, or graphs.

Every day, during the collection of data, each completed questionnaire was checked for consistency and completeness by investigators. Through the course of data collections, a regular discussion was held in between group members in which problematic issues arising during data collection, and incomplete data was cleared.

\section{Results}

3.1. Sociodemographic Characteristics. The response rate becomes $95.53 \%$. Mean age $( \pm S D)$ of study participants become $27.22 \pm 4.82$ years. Among the participants, most are male $(77.6 \%)$, and the young were aged twenty-seven or below $(52.8 \%)$. One-third of respondents attend primary education, $33.6 \%$. The mean monthly income of a respondent is 2046.06 ETB. One hundred and ninety-seven (92.1\%) describes their employment status as permanent (Table 1).

3.2. Meat Handling Practice Responses of Participants. Most of the respondents are good at safety practices such as washing hands after disposing of garbage and before handling meat (91.6\% \& 92.1\%). However, they are less serious about washing hands after visiting the toilet; and to the worst, after sneezing \& coughing. Nearly half of them remove personal stuff while processing meat (51.4\%). A considerable proportion of participant does not refrain from handling meat while felt ill or having cut, wounds in their hands. The lowest right answer is given to the practice of using gloves (9.8\%) (Table 2).

3.3. Factors Associated with Meat Handling Practice. Level of practice becomes $66.4 \%$ [95\% CI: $(59.8,72.4)]$. Sex, religion experience, attitude, and practice were found significantly 
TABLE 1: Sociodemographic and behavioral characteristics of meat handlers working at butcher shops at Gondar town, April 2019 $(n=214)$.

\begin{tabular}{|c|c|c|}
\hline \multirow{2}{*}{ Variables } & \multicolumn{2}{|c|}{ Meat handlers } \\
\hline & Frequency & Percentage \\
\hline \multicolumn{3}{|l|}{ Sex } \\
\hline Male & 166 & 77.6 \\
\hline Female & 48 & 22.4 \\
\hline \multicolumn{3}{|l|}{ Age } \\
\hline$\leq 27$ & 113 & 52.8 \\
\hline$\geq 28$ & 101 & 47.2 \\
\hline \multicolumn{3}{|l|}{ Religion } \\
\hline Orthodox & 175 & 81.8 \\
\hline Others ${ }^{¥}$ & 39 & 18.2 \\
\hline \multicolumn{3}{|l|}{ Educational status } \\
\hline Unable to read and write & 19 & 8.9 \\
\hline Primary education (1-8) & 72 & 33.6 \\
\hline Secondary education (9-12) & 76 & 35.5 \\
\hline Higher education $(12+)$ & 47 & 22.0 \\
\hline \multicolumn{3}{|l|}{ Marital status } \\
\hline Married & 94 & 43.9 \\
\hline Single & 120 & 56.1 \\
\hline \multicolumn{3}{|l|}{ Income } \\
\hline 1000 and below & 46 & 21.5 \\
\hline $1001-2000$ & 101 & 47.2 \\
\hline $2001-3000$ & 31 & 14.5 \\
\hline 3001 and above & 36 & 16.8 \\
\hline \multicolumn{3}{|l|}{ Working condition } \\
\hline Permanent & 197 & 92.1 \\
\hline Contract/daily & 17 & 7.9 \\
\hline \multicolumn{3}{|l|}{ Knowledge } \\
\hline Poor & 108 & 50.5 \\
\hline Good & 106 & 49.5 \\
\hline \multicolumn{3}{|l|}{ Attitude } \\
\hline Poor & 41 & 19.2 \\
\hline Good & 173 & 80.8 \\
\hline
\end{tabular}

NB: ${ }^{¥}$ Muslim \& protestant.

associated with a value of $p$ value of $<0.2$ in bivariable logistic regression. While running into multivariable logistic, attitude and knowledge remains significant (Table 3 ).

\section{Discussion}

Maintenance of proper hygienic practices is at the top of the agenda in food \& drinking establishments, while handling is essential to provide fresh and healthy meat for public consumption [36]. In this cross-sectional study, we try to explore the level of good practice among butcher houses with potential determinants. The level of good practice of meat handling among workers in butcher houses becomes $66.4 \%$. This is the reality of developing countries like Ethiopia, where either awareness or lack of essential facilities does not make practice as expected. It is similar to food handlers in food establishments in Dessie (72\%) [37], Dangila (52.5\%) [38], Ethiopia.

It is lower than some of the hygiene behavior-related responses like washing hands after using the toilet in Malaysian canteens 75.4\% [39], Ethio-Somalia butchers $86.8 \%$ [20]; after handling garbage (94.3\%), smoking and sneezing 95.4\%, respectively [21], refraining from dining $(70.7 \%)$ or smoking $(96.8 \%)$ at the workplace [19]. Similarly, utilization of PPE-related practices include hairnet use in Saudi 96.6\% [21] and wearing an apron in Iran 95.9\% [19]. The discrepancy will be due to sociodevelopment, enforcement capacity, and even Islamic religious routines that influence personal hygiene conducts due to their stringent rules about the purity of foods in food handling [40].

It is higher than mothers in Debarq town [41], public food establishments in northwest Ethiopia [42], and Araba minch [43]. Moreover,it is higher, compared to unaggregated responses like handle money while receiving customers in Gondar butcher shops (45.3\%) [9], wearing an apron during work $(38.3 \%)$ [7]. The difference can be implicated to the very variation in analysis plan, cutoff point, operational definition, and scope of research and tool composition.

Those workers with a good level of knowledge concerning food safety have odds of 2 times more engaged in good practices. It is accepted that knowledge alone is insufficient to trigger preventive practices, and some mechanism is needed to motivate action and generate positive attitudes [22]. This is in line with Malaysian food handlers $(p=0.041)$ [44], eastern Ethiopia $(\mathrm{AOR}=10.4,95 \% \mathrm{CI}:(4.6$, 23.81)) [45]. The educational level appears to affect the attitude $(p=0.001)$ and practice $(p=0.009)$ of the participants [46] that shows an indirect influence of knowledge to practice; having an educational level plays an undisputed role in shaping the knowledge of food handlers.

However, a significant negative association was reported between knowledge and practices $(p=0.04)$ in Iran meat processing industry [19]. This does not necessarily imply that nothing is contributed by knowledge; rather, it indicates some impediments that prevent the conversion of knowledge into attitude and gradually to tangible practices. These bottlenecks can be like unavailability of bathroom facilities [45] and intermittent water supply.

Attitude manifests itself as the strongest factor that steers the level of practice of food handlers. The odds of exercising a good level of meat handling practice are 4.5 times among those with a good attitude towards safe food handling practice. Attitude is the proximal factor that determines the translation into observable action. This supported with previous investigations like public food handling establishments in northwest Ethiopia (AOR $=1.97,95 \%$ CI 1.04, 3.72) [42], Malaysian food handlers $(p=0.041)$ [44], and Debarq mothers (AOR 3.67, 95\% CI: 2.27, 5.94) [41]. A discordant result was observed in some literature; for example, Iranian study came up with a significant negative correlation between attitudes and practices ( $r s=\ldots 0.27$, $p=0.009$ ) [19]. This may be attributed to scenarios in which the attitudinal change is just due to social desirability bias.

The study is in short of incorporating observations during meat handling operations. Thus, it is a result of a selfreport of the meat handlers. Moreover, scarcity of the related literature gives a hard time to compare results with aggregate findings rather than resorting to using specific response questions. It is crucial to admit that having a relatively small sample size will have a role in overall generalizability. 
TABLE 2: Meat handling practice of meat handlers at butcher shops at Gondar town, April $2019(n=214)$.

\begin{tabular}{|c|c|c|}
\hline \multirow{2}{*}{ Practice questions } & \multicolumn{2}{|c|}{ Responses $n(\%)$} \\
\hline & Right & Wrong \\
\hline Do you eat or drink at your workplace? & $107(50.0)$ & $107(50.0)$ \\
\hline Do you smoke inside meat processing areas? & $194(90.7)$ & $20(9.3)$ \\
\hline Do you use gloves while handling meat? & $20(9.3)$ & $194(90.7)$ \\
\hline Do you handle money while processing meat? & $21(9.8)$ & $193(90.2)$ \\
\hline Do you wash your hands before and after handling meat? & $197(92.1)$ & $17(7.9)$ \\
\hline Do you wash hands after handling waste/garbage? & $196(91.6)$ & $18(8.4)$ \\
\hline Do you wash hands after using the toilet? & $192(89.7)$ & $22(10.3)$ \\
\hline Do you wash your hand after smoking, sneezing, or coughing? & $137(64.0)$ & $77(36.0)$ \\
\hline Do you wear an apron while working? & $101(47.2)$ & $113(52.8)$ \\
\hline Do you wash your aprons after each day's work? & $175(81.8)$ & $39(18.2)$ \\
\hline Do you wear a mask while working? & $81(37.9)$ & $133(62.1)$ \\
\hline Do you wear a hairnet or a cap while working? & $198(92.5)$ & $16(7.5)$ \\
\hline Do you use and paint nail polish when handling meat. & $190(88.8)$ & $24(11.2)$ \\
\hline Do you properly clean the meat storage area before storing new products? & $199(93.0)$ & $15(7.0)$ \\
\hline Do you use the sanitizer when washing service utensils (knives, hooks, and cutting boards)? & $160(74.8)$ & $54(25.2)$ \\
\hline Do you replace knives or sterilize them after each meat processing? & $135(63.1)$ & $79(36.9)$ \\
\hline Do you remove your work equipment when using the toilets? & $188(87.9)$ & $26(12.1)$ \\
\hline Do you remove your personal stuff such as rings, necklaces, watch, etc. while processing meat & $110(51.4)$ & $104(48.6)$ \\
\hline Do you handle/process meat when you are ill? & $116(54.2)$ & $98(45.8)$ \\
\hline Do you handle/process meat when you have cuts, wounds, bruises, or injuries on your hands? & $140(65.4)$ & $74(34.6)$ \\
\hline
\end{tabular}

TABLE 3: Bivariable and multivariable logistic regression of factors associated with the practice of meat handlers working at butcher shops at Gondar town, April $2019(n=214)$.

\begin{tabular}{|c|c|c|c|c|}
\hline \multirow{2}{*}{ Variables } & \multicolumn{2}{|c|}{ Meat safety practice } & \multirow{2}{*}{ COR $(95 \%$ CI $)$} & \multirow{2}{*}{ AOR (95\% CI) } \\
\hline & Good & Poor & & \\
\hline \multicolumn{5}{|l|}{ Experiences } \\
\hline 2 and below & 65 & 29 & 1 & 1 \\
\hline $3-4$ & 52 & 25 & $0.92(0.48,1.77)$ & $0.93(0.46,1.87)$ \\
\hline 5 and above & 25 & 18 & $0.62(0.29,1.30)$ & $0.62(0.28,1.41)$ \\
\hline \multicolumn{5}{|l|}{ Sex } \\
\hline Male & 106 & 60 & 1 & 1 \\
\hline Female & 36 & 12 & $1.69(0.82,3.51)$ & $2.03(0.91,4.50)$ \\
\hline \multicolumn{5}{|l|}{ Religion } \\
\hline Orthodox & 120 & 55 & $1.68(0.83,3.42)$ & $1.53(0.71,3.33)$ \\
\hline Others ${ }^{¥}$ & 22 & 17 & 1 & 1 \\
\hline \multicolumn{5}{|l|}{ Attitude } \\
\hline Good & 127 & 46 & $4.78(2.33,9.82)^{* * *}$ & $4.45(2.09,9.43)^{* * *}$ \\
\hline Poor & 15 & 26 & 1 & 1 \\
\hline \multicolumn{5}{|l|}{ Knowledge } \\
\hline Good & 80 & 26 & $2.28(1.27,4.04)^{* *}$ & $2.04(1.09,3.82)^{*}$ \\
\hline Poor & 62 & 46 & 1 & 1 \\
\hline
\end{tabular}

NB: significant at $p^{*}=0.05,{ }^{* *}=0.006,{ }^{* * *}=0.0001$ and 1 is the reference; ${ }^{*}=$ Muslim and protestant.

\section{Conclusion}

More than half the number of meat handlers in Gondar town had a good level of self-reported food safety practice. In general, their food safety practice level was found comparable with earlier studies and by far lower than universally expected a higher standard of practice. There was no significant relationship with the level of food safety practice and some demographic variables (age, educational level, income, and experience). Knowledge and attitude were found as the factors having a stronger significant association with the food safety practice of meat handlers. Therefore, food safety knowledge and positive attitude of meat handlers, through frequent mentoring-based supervisory work, facilitating the availability of sanitation facilities are important interventions to enhance their level of food safety practice.

\section{Abbreviations}

AOR: Adjusted odds ratio

CI: Confidence interval

COR: Crude odds ratio

ETB: Ethiopian Birr

Km: Kilometer

SD: Standard deviation

SPSS: Statistical package for social sciences

PEP: Personal protective equipment. 


\section{Data Availability}

The data used to support the findings of this study are available from the corresponding author upon a reasonable request.

\section{Ethical Approval}

Ethical clearance was obtained from the department of EOHS. Similarly, the respondent was informed about the purpose of the study, and verbal informed consent was obtained. Any worker was neither forced to participate against their will nor paid for their participation, and data collectors tell the study is nothing to do with regulatory or compliance undertakings.

\section{Conflicts of Interest}

All of the authors do not have any conflicting interest in the manuscript.

\section{Authors' Contributions}

Dawit Getachew Yenealem conceived the research projects, designed and modified the tool, and involved in supervising the daily collection of data. Walelegn Worku Yallew participated in the interpretation of the results and prepared the manuscripts. Shafi Abdulmajid was involved in analyses of the data and overseeing data collection. All authors read and approved the manuscript.

\section{Acknowledgments}

The authors are indebted to acknowledge data collectors, field supervisors, study participants, University of Gondar, and Gondar town administrators for their unreserved contributions to the success of this study; otherwise, it would have been in jeopardy. The University of Gondar covered questionnaire duplication.

\section{References}

[1] I. Soriyi, H. Agbogli, and J. Dongdem, "A pilot microbial assessment of beef sold in the Ashaiman market, a suburb of Accra, Ghana," African Journal of Food, Agriculture, Nutrition and Development, vol. 8, no. 1, pp. 91-103, 2008.

[2] A. Bersisa, D. Tulu, and C. Negera, "Investigation of bacteriological quality of meat from abattoir and butcher shops in Bishoftu, Central Ethiopia," International Journal of Microbiology, vol. 2019, Article ID 6416803, 8 pages, 2019.

[3] B. Akinyera, A. A. Maimadu, O. C. Akinsulie, M. P. Olabode, J. A. Sabo, and O. H. Osemeke, "Microbial loads of beef and hygienic practice of butchers in Jos municipal abattoir," Asian Journal of Research in Animal and Veterinary Sciences, vol. 1, no. 4, pp. 1-9, 2018.

[4] A. Abdullahi, A. Hassan, N. Kadarman, A. Saleh, Y. B. Shuaibu, and P. L. Lua, "Food safety knowledge, attitude, and practice toward compliance with abattoir laws among the abattoir workers in Malaysia," International Journal of General Medicine, vol. 9, p. 79, 2016.

[5] F. Nyamakwere, V. Muchenje, B. Mushonga, E. Kandiwa, M. Makepe, and G. Mutero, Evaluation of Meat Safety
Knowledge, Attitudes and Practices Among Slaughter House Workers of Amathole District in Eastern Cape Province, South Africa, UNAM, Windhoek, Namibia, 2017.

[6] T. Tefera and G. Mebrie, "Prevalence and predictors of intestinal parasites among food handlers in Yebu town, southwest Ethiopia," PLoS One, vol. 9, no. 10, Article ID e110621, 2014.

[7] F. Akabanda, E. H. Hlortsi, and J. Owusu-Kwarteng, "Food safety knowledge, attitudes and practices of institutional foodhandlers in Ghana," BMC Public Health, vol. 17, no. 1, p. 40, 2017.

[8] P. Lamuka, S. Chepkemoi, G. Ooko Abong, and J. Wafula Matofari, "Sanitation and hygiene meat handling practices in small and medium enterprise butcheries in Kenya-case study of Nairobi and Isiolo counties," Internet Journal of Food Safety, vol. 17, pp. 64-74, 2015.

[9] W. Birhanu, S. Weldegebrie, G. Bassazin, F. Mitku, L. Birku, and M. Tadesse, "Assesment of microbiological quality and meat handling practices in butcher shops and abattoir found in Gondar town, Ethiopia," International Journal of Microbiological Research, vol. 8, no. 2, pp. 59-68, 2017.

[10] WHO, WHO's First Ever Global Estimates of Foodborne Diseases Find Children under 5 Account for Almost One Third of Deaths, WHO, Geneva, Switzerland, 2015, https://www. who.int/news-room/detail/03-12-2015-who-s-first-everglobal-estimates-of-foodborne-diseases-find-children-under5-account-for-almost-one-third-of-deaths.

[11] WHO, WHO Estimates of the Global Burden of Foodborne Diseases: Foodborne Disease Burden Epidemiology Reference Group 2007-2015, World Health Organization, Geneva, Switzerland, 2015.

[12] M. J. Mangen, I. H. M. Friesema, R. Pijnacker, L. Mughini Gras, and W. van Pelt, Disease Burden of Food-Related Pathogens in the Netherlands, RIVM, Bilthoven, Netherlands, 2018.

[13] WorldBank, "Food-borne illnesses cost US\$ 110 billion per year in low- and middle-income countries," WorldBank, Washington, DC, USA, 2018, https://www.worldbank.org/en/ news/press-release/2018/10/23/food-borne-illnesses-cost-us110-billion-per-year-in-low-and-middle-income-countries.

[14] F. Adugna, M. Pal, and G. Girmay, "Prevalence and antibiogram assessment of Staphylococcus aureus in beef at municipal abattoir and butcher shops in Addis Ababa, Ethiopia," BioMed Research International, vol. 2018, Article ID 5017685, 7 pages, 2018.

[15] L. S. Soares, R. C. C. Almeida, E. S. Cerqueira, J. S. Carvalho, and I. L. Nunes, "Knowledge, attitudes and practices in food safety and the presence of coagulase-positive staphylococci on hands of food handlers in the schools of Camaçari, Brazil," Food Control, vol. 27, no. 1, pp. 206-213, 2012.

[16] WHO, "Food safety key facts," WHO, Geneva, Switzerland, 2019, https://www.who.int/news-room/fact-sheets/detail/ food-safety.

[17] I. F. Angelillo, N. M. A. Viggiani, R. M. Greco, and D. Rito, "HACCP and food hygiene in hospitals knowledge, attitudes, and practices of food-services staff in Calabria, Italy," Infection Control \& Hospital Epidemiology, vol. 22, no. 6, pp. 363-369, 2001.

[18] N. A. Sani and O. N. Siow, "Knowledge, attitudes and practices of food handlers on food safety in food service operations at the Universiti Kebangsaan Malaysia," Food Control, vol. 37, pp. 210-217, 2014.

[19] M. Ansari-Lari, S. Soodbakhsh, and L. Lakzadeh, "Knowledge, attitudes and practices of workers on food hygienic practices 
in meat processing plants in Fars, Iran," Food Control, vol. 21, no. 3, pp. 260-263, 2010.

[20] H. A. Tegegne and H. Phyo, "Food safety knowledge, attitude and practices of meat handler in abattoir and retail meat shops of Jigjiga town, Ethiopia," Journal of Preventive Medicine and Hygiene, vol. 58, no. 4, p. E320, 2017.

[21] N. A. Al-Shabib, S. H. Mosilhey, and F. M. Husain, "Crosssectional study on food safety knowledge, attitude and practices of male food handlers employed in restaurants of King Saud University, Saudi Arabia," Food Control, vol. 59, pp. 212-217, 2016.

[22] M. B. Egan, M. M. Raats, S. M. Grubb et al., "A review of food safety and food hygiene training studies in the commercial sector," Food Control, vol. 18, no. 10, pp. 1180-1190, 2007.

[23] EFSA, "The community summary report on trends and sources of zoonoses, zoonotic agents and food-borne outbreaks in the European Union in 2008," EFSA Journal, vol. 8, no. 1, p. 1496, 2010.

[24] H. K. Adesokan and A. Raji, "Safe meat-handling knowledge, attitudes and practices of private and government meat processing plants' workers: implications for future policy," Journal of Preventive Medicine and Hygiene, vol. 55, no. 1, p. 10, 2014.

[25] L. H. Gould, I. Rosenblum, D. Nicholas, Q. Phan, and T. F. Jones, "Contributing factors in restaurant-associated foodborne disease outbreaks, FoodNet sites, 2006 and 2007," Journal of Food Protection, vol. 76, no. 11, pp. 1824-1828, 2013.

[26] J. F. R. Lues and I. Van Tonder, "The occurrence of indicator bacteria on hands and aprons of food handlers in the delicatessen sections of a retail group," Food Control, vol. 18, no. 4, pp. 326-332, 2007.

[27] M. Haileselassie, H. Taddele, K. Adhana, and S. Kalayou, "Food safety knowledge and practices of abattoir and butchery shops and the microbial profile of meat in Mekelle city, Ethiopia," Asian Pacific Journal of Tropical Biomedicine, vol. 3, no. 5, pp. 407-412, 2013.

[28] A. K. C. Campos, Â. M. S. Cardonha, L. B. G. Pinheiro, N. R. Ferreira, P. R. M. d. Azevedo, and T. L. M. Stamford, "Assessment of personal hygiene and practices of food handlers in municipal public schools of Natal, Brazil," Food Control, vol. 20, no. 9, pp. 807-810, 2009.

[29] L. Sharif and T. Al-Malki, "Knowledge, attitude and practice of Taif University students on food poisoning," Food Control, vol. 21, no. 1, pp. 55-60, 2010.

[30] C. Jianu and I. Goleț, "Knowledge of food safety and hygiene and personal hygiene practices among meat handlers operating in western Romania," Food Control, vol. 42, pp. 214-219, 2014.

[31] A. K. Jones, "Estimating the prevalence of food risk increasing behaviours in UK kitchens," PLoS One, vol. 12, no. 6, Article ID e0175816, 2017.

[32] F. Adzitey, G. Teye, and M. Dinko, Pre and Post-Slaughter Animal Handling by Butchers in the Bawku Municipality of the Upper East Region of Ghana, Livestock Research for Rural Development, Cali, Colombia, 2011.

[33] S. Birhanu and S. Menda, "Hygienic handling and processing of raw beef meat at slaughter houses and meat stalls in Gojjam area, Ethiopia," International Journal of Veterinary Health Science \& Research, vol. 5, no. 8, pp. 213-218, 2017.

[34] S. Seleshe, C. Jo, and M. Lee, "Meat consumption culture in Ethiopia," Korean Journal for Food Science of Animal Resources, vol. 34, no. 1, pp. 7-13, 2014.
[35] Federal Demographic Republic of Ethiopia, Population Projection of Ethiopia for All Regions at Wereda Level from 2014-2017, Federal Demographic Republic of Ethiopia, Addis Ababa, Ethiopia, 2013.

[36] G. Khanal and S. Poudel, "Factors associated with meat safety knowledge and practices among butchers of Ratnanagar municipality, Chitwan, Nepal: a cross-sectional study," Asia Pacific Journal of Public Health, vol. 29, no. 8, pp. 683-691, 2017.

[37] M. Adane, B. Teka, Y. Gismu, G. Halefom, and M. Ademe, "Food hygiene and safety measures among food handlers in street food shops and food establishments of Dessie town, Ethiopia: a community-based cross-sectional study," PLoS One, vol. 13, no. 5, Article ID e0196919, 2018.

[38] A. G. Tessema, K. A. Gelaye, and D. H. Chercos, "Factors affecting food handling Practices among food handlers of Dangila town food and drink establishments, North West Ethiopia," BMC Public Health, vol. 14, no. 1, p. 571, 2014.

[39] S. O. Nee and N. A. Sani, "Assessment of knowledge, attitudes and practices (KAP) among food handlers at residential colleges and canteen regarding food safety," Sains Malaysiana, vol. 40, no. 4, pp. 403-410, 2011.

[40] S. I. A. Rahim, S. K. M. Mansor, M. A. Yakob, and N. Ismail, "Food safety, sanitation and personal hygiene in food handling: an overview from Islamic perspective," International Journal of Civil Engineering and Technology (IJCIET), vol. 9, no. 9, pp. 1524-1530, 2018.

[41] H. Dagne, R. P. Raju, Z. Andualem, T. Hagos, and K. Addis, "Food safety practice and its associated factors among mothers in Debarq town, northwest Ethiopia: communitybased cross-sectional study," BioMed Research International, vol. 2019, Article ID 1549131, 8 pages, 2019.

[42] F. A. Chekol, M. F. Melak, A. K. Belew, and E. G. Zeleke, "Food handling practice and associated factors among food handlers in public food establishments, northwest Ethiopia," BMC Research Notes, vol. 12, no. 1, p. 20, 2019.

[43] D. Legesse, M. Tilahun, E. Agedew, and D. Haftu, "Food handling practices and associated factors among food handlers in Arba Minch town public food establishments in Gamo Gofa Zone, Southern Ethiopia," Epidemiology: Open Access, vol. 7, no. 2, pp. 2161-1165, 2017.

[44] N.-A. Abdul-Mutalib, M.-F. Abdul-Rashid, S. Mustafa, S. Amin-Nordin, R. A. Hamat, and M. Osman, "Knowledge, attitude and practices regarding food hygiene and sanitation of food handlers in Kuala Pilah, Malaysia," Food Control, vol. 27, no. 2, pp. 289-293, 2012.

[45] N. Baraki and G. Egata, Food Safety Practice and Associated Factors among Food Handlers in Public Food Establishments of Godey Town, Somali Region, Eastern Ethiopia, Haramaya University, Dire Dawa, Ethiopia, 2018.

[46] H. Adetunji, "Knowledge, attitude, and practice (KAP) of personal hygiene among food handlers in the south region of Makkah, Saudi Arabia," International Journal of Medical Research \& Health Sciences, vol. 7, no. 5, pp. 96-102, 2018. 